\title{
Evaluating Police Administration Performance Using Data Envelopment Analysis
}

\author{
Tien-Chin Wang, ${ }^{1,}$, Bi-Chao Lee ${ }^{1,2}$ \\ ${ }^{1}$ Dept. of International Business, National Kaohsiung University of Applied Sciences, Kaohsiung, Taiwan \\ ${ }^{2}$ Kaohsiung City Police Department, Kaohsiung, Taiwan
}

\section{Email address:}

tcwang@kuas.edu.tw (Tien-Chin Wang),1103405113@gm.kuas.edu.tw (Bi-Chao Lee)

${ }^{*}$ Corresponding author

\section{To cite this article:}

Tien-Chin Wang, Bi-Chao Lee. Evaluating Police Administration Performance Using Data Envelopment Analysis. Science Innovation. Vol. 5, No. 4, 2017, pp. 194-201. doi: 10.11648/j.si.20170504.11

Received: March 30, 2017; Accepted: April 22, 2017; Published: May 4, 2017

\begin{abstract}
Police work contains many aspects, each of which was held to specific performance standards. Past literature has investigated individual cases and made selective comparisons, yet is lacking in overall work analyses. Police authorities must be able to assess the work performances of each police station to ensure the balance of investments and returns while investigating overall work performance, rather than solely looking at individual cases. This study used objective analysis to evaluate the 2014 work performances of Kaohsiung City Police Department police stations (Taiwan) in 00 Precinct in order to determine the quality of work performed so that the heads of each unit can improve any insufficiencies in accordance with objectives and improve the competitiveness of police work. The Data Envelopment Analysis (DEA) was used to arrange these indicators according to 00 Precinct's police stations (DMU) relative work performance. Nine police stations (A-I) and seven indicators were included in this study to assess the work performances of each station. The population in the area of jurisdiction were used to a baseline for normalization of indicators. The results indicated that among the nine police stations, H,F, E and I stations had the better relative performance in the 00 Precinct that analyzed by Deap version 2.1. If the performance management system (PBM) still be used for Performance Evaluation in police organization to set performance goals. This proposal can be made a number of assessment criteria to scheduling the degree of complexity of the police stations, than to order the stations of the set target value should be reached so that units performance measurement benchmarks and more realistically.
\end{abstract}

Keywords: Police-to-Population Ratio, Performance Evaluation, Delphi, Data Envelopment Analysis (DEA)

\section{以DEA評估警政績效之研究}

\section{王天津 $^{1 *}$, 李碧昭 ${ }^{1,2}$}

${ }^{1}$ 高雄應用科技大學國企系, 高雄市, 台灣

高雄市政府警察局, 高雄市, 台灣

邮箱

tcwang@kuas. edu. tw（王天津），1103405113@gm. kuas. edu. tw（李碧昭）

摘要：警察工作績效要求很多, 因應治安需要執行各種專案, 由專案或選擇性幾個面向做績效評估, 但如何評量一個 單位的整體工作績效, 以確保符合投入與產出的均衡, 值得探討。本研究嚐試以DEA的分析方法, 對高雄市政府警察局 00分局各派出所, 以2014年為基礎, 參考幾項重要指標, 再以相對績效評量的資料包絡分析法 (DEA), 找出各派出所績 效最大質, 再依序檢視各所的相對績效。以A I等9個派出所為研究樣本, 警力數、用油預算為投入項, 全般刑案發生 數、全般刑案破獲數、全般車禍發生數、交通違規取締數、110受理案件數等五項為產出指標, 研究結果顯示, 受評估 
的9家派出所中, H, F, E和I樣本為00分局執行效率相對最佳的派出所。另依德菲 (Delphi)法計算出5項產出的權值, 評估結果與原結果做一個比較, 以判幽評估結果的穩定性。若警察機關對於績效評估仍需延襲使用業績管理系統 (PBM), 即需先設定績效目標, 本文建議可提出數項評估準則後, 再以本法評估各受評單位的投入產出目標值, 再依序設定各 單位應達成的目標值，使各單位的績效評核基準，更符合實際。

关键词：警力數，績效評估，德菲法(Delphi)，資料包絡分析法 (DEA)

\section{1. 引言}

警察機關對各項工作績效要求很多, 因應治安需要執 行各種專案，由專案或選擇性幾個面向做績效評估，但缺 乏一個單位的整體工作績效評量, 曾有研究者針對警察機 關以Data Envelopment Analysis (DEA) 的方法做績效評估, 但其產出的選擇偏向犯罪偵防的成效, 且其選擇以大樣本 為主 (刑台平, 2001)（紀欣儀，2007）（張國讚，2013）, 只評估優劣但未提出改善方向, 本文以為警察的工作績效 評估, 應包含治安、交通、及服務三個面向, 並應針對所 評估對象能有較具體的改善建議, 故嚐試以DEA的分析方 法, 並以最基層且具有決策權及同質性的 (吳濟華、何柏 正, 2008) 派出所為DMU, 復以台灣高雄市政府警察局00 分局各派出所2015年各項工作績效為基礎, 選擇重要指標, 分析出各派出所相對效率, 並對不足之處, 提供改善意見, 並期望透過工作績效指標及評核工具探討, 找出適合警察 機關之工作績效優劣評核方式, 使績效評核更符合基層單 位在工作上的努力的展現, 作為今後警政工作績效評核指 標的設定、評量工具選擇的參考, 以提高警政工作的競爭 力, 並確保符合投入與產出的均衡。

\section{2. 文獻探討}

\section{1. 警政工作的內涵}

警察的任務為依法維持公共秩序，保護社會安全，防 止一切危害, 促進人民福利。在傳統的概念, 係為安寧的 維護者(維持秩序)。縱然現代警察機關已限縮了原有的警 察作用 (戶政、消防、入出國境、海域保安移出), 被界定 在犯罪控制的力量, 但仍是代表政府公權力, 處理社會及 涉法案件的主要官員。

績效 (Performance) 是對組織目標達成程度的衡量, 且是組織成效的重要指標 [1], 但公務部門提供的商品和 服務, 很難找到合適的評估尺度。公務部門向來對於績效 評估大都使用業績管理系統 (PBM), 即先設定績效目標、 律定期間, 再看達成率如何, 做為績效評估方式。績效評 估工具應具有 5 種特質：具有明確性、具有綜合性、直接 反映目標、可運算性及可理解性等 [2]。並需要有關鍵績 效指標 (KPIs) 為依據, 量的指標容易評量運算, 質的指標 則比較難於掌握 [3]。台灣對警政工作的績效評估指標, 大部分是單項的。但警察的任務, 舉2014年度台灣內政部 施政計畫為例, 警察的工作項目有: 加強犯罪預防, …… 保護婦幼安全, ……取締惡性交通違規, ……, 提升犯 罪偵查能量; ……提升治安維護能力等, 項目很多。僅
以一小部分工作績效來決定該單位的工作成效, 無法得其 全貌。本文歸納後, 以2015年春安工作項目之三大面向為 產出重要指標: 1. 治安 (以全般刑案發生數、全般刑案破 獲數)、2. 交通(以全般車禍發生數、交通違規取締數)、 3. 服務 (以 110 受理案件數) 再以相關數據為正規化分母, 求比率, 為評估準則; 而投入則以警力數、用油的決算數 為評估準則, 以資料包絡分析法 (DEA), 來評定各單位工 作績效, 得到相對效率優劣指標, 並對相對無效率之單位, 找出應改進之方向, 以檢討策進, 發揮研究之實際價值。

\section{2. Data Envelopment Analysis (DEA)}

生產過程可以定義為一組資源由生產單位轉變為理 想結果的過程。效率即是用來衡量, 生產單位在利用其資 源的過程中, 成果方面的進展如何。DEA 源於Farre11 （1957）提出以「非預設生產函數」代替常用的「預設函 數」來推估效率值的概念, 利用數學規劃技巧求出效率邊 界, 亦即效率生產函數, 傳統上使用決策單元（decision making unit, DMU或決策單位) 為每個單位的分析基礎, Data Envelopment Analysis (DEA) 的名稱乃由 Charnes, Cooper, and Rhodes (1978) 在論文中定義, 是由技術 效率觀念而發展建立數學規劃模式的效率評估模式, 其主 要發展出 $\mathrm{CCR}$ 及 $\mathrm{BCC}$ 兩種基本模式, 是一種結合質性 (qualitative) 及量性 (quantitative) 的績效評估方法。 各式各樣的 DEA 模型, 在確定n個決策單元如何表示最佳 的結果, 稱為實證生產函數或有效前沿的包絡面。評估結 果聚集在包絡面的表面的單位被視為有效率的單位, 在包 絡面以下的單位, 則屬相對無效率。DEA模型可提供多個 投入及多個產出的綜合分析和測量每個 DMU 的相對效率。 此方法是採用相對比較之觀念, 接受評估的DMU互相比較, 然後利用實際觀察點與此邊界的相對位置關係, 求出技術 效率（technical efficiency, TE）。再考慮投入要素價 格比, 可測得價格效率 (price efficiency) 或稱為分配 效率（Allocative Efficiency, AE）; 總效率（overall efficiency, OE) 則為此二者的乘積。技術效率所講求的 是在現有技術上, 有效利用生產要素, 以生產最大產量之 意; 價格效率則為在既定的技術效率及要素價格比率下, 使投入的要素組合之成本為最低的生產方法（劉春初, 1998）。DEA 模式計算的效率衡量觀念, 是所謂的相對效 率 (relative efficiency), 將被評估單位 (DMU) 區分為有 效率 (efficiency) 與無效率 (inefficiency) 兩種。即在各 接受評估單位最有利情況下作最佳選擇, 各單位之效率值 須介於 0 與 1 之間，而最具效率的決策單位（DMU）其效率 值為 1 。也就是說, CCR 模式希望在固定規模報酬 (CRS) 下 所有DMU的效率值皆小於或等於 1 的情況下，予某一DMU最 
有利之要素加權值, 儘量提昇該DMU之效率值。Banker等 人於 1984 年將CCR模式, 修正為變動規模報酬 (VRS) 的假設 下, 衡量決策單位之相對效率, 即BCC模式為DEA第2種模 式。

DEA可用來評估非營利機構或政府機構的營運效率。 諸如醫院、學校、警察分局、 $\cdots \cdots$, 都曾用此法進行評估 (張錫峰、周齊武)。

\section{2. 1. 固定規模報酬模式}

CRS也就是每一單位投入可得產出量是固定的, 不會 因規模大小而改變DUMk效率定義如下:

$$
\begin{gathered}
E_{k}=\frac{\sum_{j=1}^{n} u_{j}^{k} Y_{j}^{k}}{\sum_{i=1}^{m} v_{i}^{k} X_{i}^{k}} X_{i}^{k}, Y_{j}^{k} \geq 0, i=1, \ldots, m ; j=1, \ldots, n \\
v_{i}^{k}, u_{j}^{k} \geq 0, i=1, \ldots, m ; j=1, \ldots, n
\end{gathered}
$$

$\mathrm{DEA}$ 線性規劃模式乃是以一個決策單位 $\mathrm{DMU}_{\mathrm{k}}$ 的效率 $E_{k}$ 最大化為目標式, 尋找對 $\mathrm{DMU}_{\mathrm{k}}$ 最有利的投入項權重組合, 以及產出項權重組合, 使得 $\mathrm{E}_{\mathrm{k}}$ 達最大值, 所有 $\mathrm{DMU}_{\mathrm{k}}$ 的效 率 $\mathrm{E}_{\mathrm{r}} \leqq 1$ 。決策變數: 權重 $v_{i}^{k}, u_{j}^{k}$ 為待決定之未知數。

$$
\operatorname{Max} E_{k}=\frac{\sum_{j=1}^{n} u_{j}^{k} Y_{j}^{k}}{\sum_{i=1}^{m} v_{i}^{k} X_{i}^{k}}
$$

$$
\begin{gathered}
\text { 限制式 } \sum_{i=1}^{m} v_{i}^{k} X_{i}^{k}=1 \\
\text { s.t. } \frac{\sum_{j=1}^{n} u_{j}^{k} Y_{j}^{r}}{\sum_{i=1}^{m} v_{i}^{k} X_{i}^{k r}} \leq 1, r=1,2, \ldots, R \\
u_{j}^{k} \geq \varepsilon>0, j=1,2, \ldots, n \\
v_{i}^{k} \geq \varepsilon>0, i=1,2, \ldots, m
\end{gathered}
$$

其中 $Y_{j}^{r}$ 表第 $j$ 個DMU的第 $r$ 個產出值

$\mathrm{X}_{\mathrm{i}}^{\mathrm{kr}}$ 表第 $\mathrm{j}$ 個DMU的第 $\mathrm{i}$ 個投入值

$u_{\mathrm{j}}^{\mathrm{k}}$ 表第 $\mathrm{j}$ 個DMU的第 $r$ 個產出項的加權值

$v_{i}^{k}$ 表第 $j$ 個DMU的第 $i$ 個投入項的加權值

由於模式 (1) 是分數線性規劃模式, 為一種非線性 (nonlinear) 模式, 限制 $\sum_{\mathrm{i}=1}^{\mathrm{m}} \mathrm{v}_{\mathrm{i}}^{\mathrm{k}} \mathrm{X}_{\mathrm{i}}^{\mathrm{k}}=1$ 使轉化為一個線性規 劃模式, 此即CCR模式。

\subsubsection{BCC模式}

BCC 模式將DMU是否達到有效的生產規模也納入評估, 故可同時衡量規模效率與技術效率

$$
\begin{gathered}
\operatorname{Max}_{k}=\sum_{j=1}^{n} u_{j}^{k} Y_{i}^{k}-u_{0}^{k} \\
\text { s.t } \sum_{i=1}^{m} v_{i}^{k} X_{i}^{k}=1
\end{gathered}
$$

$$
\begin{gathered}
\sum_{j=1}^{n} u_{j}^{k} Y_{i}^{r}-\sum_{i=1}^{m} v_{i}^{k} X_{i}^{r}-u_{0}^{k} \leq 0, r=1,2, \ldots, R \\
u_{j}^{k} \geq \varepsilon>0, j=1,2, \ldots, n \\
v_{i}^{k} \geq \varepsilon>0, i=1,2, \ldots, m
\end{gathered}
$$

總效率與技術效率關係: DMU未達相對有效率可能是 技術無效率或者是規模無效率, CCR模式中各個DMU生產規 模已調整至規模效率, 故求得之相對效率 $\mathrm{h}_{k}$ 為總效率 (overall efficiency); BCC模式模式中各個DMU的規模效 率並不一致, 求得之相對效率 $\mathrm{t}_{k}$ 為純技術效率, 其總效率 乃是技術效率與規模效率之乘積, CCR 模式與BCC模式均採 取「自我評估」之觀點, 也就是受評之DMU會找尋對自己 最有利之權重組合, 當一DMU之相對效率達到 1 時, 仍須觀 察該DMU被其他無效率DMU參考的次數, 被參考的次數越多 其穩定性越高, 因此, 可將評估相對效率同為1的DMU進一 步區分其效率。

\subsection{Delphi Technique法求權重}

權重方法是一種實用的決策分析技術, 在決定投入及 產出項目在整體績效上的相對重要性。在投資決策與需求 規劃等實務方面, 有著廣泛的應用領域。作權重考量時, 必須面臨決策環境及可獲得之決策資訊之限制, 而其也將 影響所選用之權重方法。如何依據實際的需要, 選擇適當 的分析工具或彰顯價值判斷相當重要 (蔡向榮, 張宏光)。 DEA分析會根據受衡量的每個DMU之投入產出項資料運算 出一組最佳的權數, 使各DMU多項產出的加權總值除以多 項投入的加權總值之比率極大, 且以此比率來定義各個 DMU之相對效率, 但權重的刻意選擇將可能造成該DMU為相 對有效率 (劉春初, 2004), 也為學者所批評, 後學者多有 以其他權重方法加以修正。求算權重的方法許多, 例如: 簡單權重、AHP、幾何平均法、數學規劃法等。又分有主 觀權重法與客觀權重法 [10]客觀權重設限法的原始數據 來自於評估矩陣的實際數據, 切斷主觀性的來源, 使權重 具有絕對的客觀性是其優點, 但也存在可能與實際相悖的 缺陷。Delphi方法是為主觀權重法之一種, The RAND Corp. 最初開發, 作為預測或預測未來的一種方式。已經被廣泛 使用在包括教育、土地利用規劃、交通、社會服務規劃、 商業, 甚至哲學等學科 [5]。是由一群在專業領域上專精 之人士或學者, 針對某項特定議題, 藉由他們豐富的實務 經驗與知識, 透過精心設計的問卷, 獲得專家群之意見。 其目的在使評鑑的標準具有權威性與說服力, 然後利用所 獲得之寶貴意見進行研究項目之定量分析。

DEA演算法在分析過程中會自動對各投入及產出項目 給予權重, 乃客觀權重法的一種, 本研究為顧及警察工作 績效與社會情勢及政治等因素相關, 尤其有文化環境的認 知, 故另再採用專家調查方法-主觀權重法, 計算評估準 則權重, 用以正規化各項數據, 為DEA評估的基礎。 


\section{3. 研究方法}

\section{1. DEAP 2.1為分析工具}

DEAP軟體為Rolf Fare, Shawna Grosskopf以及他的 團隊所撰寫來執行DEA分析的工具，而其固定規模報酬 (constant return to scale, CRS) CCR模式假設; 變動 規模報酬 (VRS) 即是BCC模式。

\section{2. 投入產出的選擇}

使用 DEA 法的第二步驟即為投入產出項之選取，所 謂投入項目即是對產出有貢獻之因子, 而產出項目則為組 織之目標, 故投入產出項目之選擇、衡量方法之決定及其 數據之正確性，為使用DEA的關鍵。本研究在選取警察分 局一年來之各項績效，各所在人員的薪資、辦公經費一般 條件都相同, 選擇有差異的條件一警力數及使用油料的決 算數為投入項; 而產出則以上述春安工作三項主軸之全年 績效為評估客觀面（以官方資料為主），應包含如表 1 。

表1 客觀面投入產出項。

\begin{tabular}{|c|c|c|}
\hline 投入/產出 & 項目名稱 & 衡量方式 \\
\hline $\mathrm{a}$ & 轄區人口數 & 作為部分指標正規化的基準 \\
\hline 投入 & 警力數 & 2014年各所警力數 \\
\hline 投入 & 車輛用油比率 (千元) & 各所使用油料決算數/1000 \\
\hline 產出 & 全般刑案發生率 & $\begin{array}{l}\text { 1-2014年各所轄區全般刑案發生數 } \\
\text { /轄區人口數 }\end{array}$ \\
\hline 產出 & 全般刑案破獲率 & $\begin{array}{l}\text { 2014年各所轄區刑案破獲數/全般 } \\
\text { 刑案發生數 }\end{array}$ \\
\hline 產出 & 全般車禍發生率 & $\begin{array}{l}\text { 1-2014年各所轄區全般車禍發生數 } \\
\text { /轄區人口數 }\end{array}$ \\
\hline 產出 & 交通違規取締數 & 2014年各所交通告發數/警力數 \\
\hline 產出 & 受理案件平均數 & $\begin{array}{l}\text { 2014年各所受理民眾報案/轄區人 } \\
\text { 口數 }\end{array}$ \\
\hline
\end{tabular}

歷來警政學者均主張警察機關績效評估制度需兼顧 查處面與預防面, 因此表 1 之 $c_{2} 、 c_{4} 、 c_{5}$ 項為查處面的的產 出項, 而 $c_{1} 、 c_{3}$ 項為預防面產出項。表1各項資料均來自 受研究之警察分局2015年度警力數、經費使用及相關刑案、 交通統計資料 (表 2 ); 人口數為該分局各派出所當年之人 口數資料; 前述預防面產出項, 例如全般刑案發生率與全 般車禍發生率為負向指標, 即資源投入越多或預防工作績
效愈好時, 各項犯罪率將越低, 由於 DEA 原始模型之同 向性要求需符合「投入增加, 產出不會降低」之假設, 唯 實務面應為投入越多發生率會降, 故為避免錯置, 因此本 研究將犯罪率及車禍率依比例變成小數再用 1 去減 (確保 不成為負值), 使成為正向指標, 即本數越小, 代表產出 越大。（刑台平, 2002)

\section{3. Delphi Technique求算主觀權重}

以所選取之評估準則設計問卷指標，並以1-10為各該 單項之重要性分數值, 由 30 人以內專家加以評分, 統計各 單項得分為 $e_{j}$, 再以總得分 (各項合計) 為分母, $e_{j}$ 為分子, 計算各評估準則 $C_{1}, C_{2}, \ldots, C_{n}$ 之權重 $e_{j}$, 表示為第 $\mathrm{j}$ 準則之 分數值, 再以總分數值為分母相除之為 $w_{j}$, 用以確保 $w_{j}$ 值 介於 0 到 1 之間。

$$
e_{j}=\sum_{i=1}^{m} x_{i}, i=1,2, \ldots, m,
$$

再計算各評估準則之權重

$$
W=\left(w_{1}, w_{2, \ldots}, w_{n}\right), w_{j}=e_{j} / \sum_{j=1}^{n} e_{j}, j=1,2, \ldots, n
$$

\section{4. 實證分析}

本研究對象為非營利且非生產單位, 故本文不在於精 確算出各DMU技術效率及規模效率, 唯DEA的模式分析乃是 將某一單位與同向性的其他單位放在一起做比較, 選出在 現有資源下, 表現最好的單位, 定其效率為百分之百, 再 將其他單位與此單位比較, 得到一個效率的百分比 (張錫 峰、周齊武, 1992), 符合目前警政機關對於績效的要求 模式, 故本研究藉由DEAP軟體運算出在同樣投入條件 (警 力數、車輛用油決算數), 各別產出資料之百分比, 再以 Delphi Technique法求主觀權重, 再分析統計出總體效率 值，為該分局各派出所之相對效率，並排訂順序。

\subsubsection{0分局2014年基本資料}

本研究因運用到車輛用油的決算數，故採前 (2014) 年的相關資料數據為基礎，並以2.1.2所述五項數據資料 $c_{1}, c_{2}, c_{3}, c_{4}, c_{5}$ 為產出。

\begin{tabular}{|c|c|c|c|c|c|c|c|c|}
\hline & 轄區人口數 & 全般刑案發生率 & 全般刑案破獲率 & 全般車禍發生率 & 交通違規取締數 & 受理案件平均數 & 警力數 & $\begin{array}{l}\text { 車輛用油 } \\
\text { 決算 (千元) }\end{array}$ \\
\hline 仁武(A) & 32636 & 689 & 438 & 1265 & 3370 & 5290 & 22 & 486867 \\
\hline 澄觀 (B) & 48162 & 656 & 366 & 1589 & 1742 & 7391 & 25 & 476588 \\
\hline 大社 (C) & 34390 & 548 & 288 & 1327 & 2662 & 5012 & 25 & 422348 \\
\hline 仁美 (D) & 16402 & 172 & 120 & 497 & 1044 & 1990 & 14 & 263767 \\
\hline 鳥松 (E) & 14724 & 364 & 262 & 556 & 1698 & 2564 & 16 & 226920 \\
\hline 大華 (F) & 11515 & 256 & 181 & 817 & 2539 & 3590 & 15 & 272558 \\
\hline 九曲 (G) & 20883 & 214 & 185 & 329 & 1550 & 1553 & 19 & 282079 \\
\hline 大樹 (H) & 13241 & 113 & 89 & 203 & 1263 & 881 & 13 & 216846 \\
\hline 溪埔 (I) & 9080 & 114 & 68 & 239 & 598 & 1225 & 13 & 247247 \\
\hline
\end{tabular}

表200分局2014年基本資料。 


\section{2. 同向性 (Isotonicity) 檢定}

同向性乃因DEA評估的是決策單位 (DMU) 的相對效率, 故DMU的選擇需具有相似性且為可計算的 (measurable), 同向性檢定係利用 Pearson 相關分析，將投入項與客觀 面之官方資料產出項間之關係, 分別予以測試, 以驗證是 否符合同向性之假設, 「即投入增加, 產出不會降低」(曾 國雄、刑台平，2002）。上述8項基本資料經以SPSS軟體運 算Pearson相關係數, 投入產出之間有中度及高度相關 (如 表3)。

表3 Pearson Correlations。

\begin{tabular}{lllllllll}
\hline & $\mathrm{a}$ & $\mathrm{c}_{1}$ & $\mathrm{c}_{2}$ & $\mathrm{c}_{3}$ & $\mathrm{c}_{4}$ & $\mathrm{c}_{5}$ & $\mathrm{~b}_{1}$ & $\mathrm{~b}_{2}$ \\
\hline $\mathrm{a}$ & 1 & & & & & & & \\
$\mathrm{c}_{1}$ & $880^{* * *}$ & 1 & & & & & & \\
$\mathrm{c}_{2}$ & $808^{* * *}$ & $975^{* *}$ & 1 & & & & & \\
$\mathrm{c}_{3}$ & $889^{* *}$ & $938^{* *}$ & $863^{* *}$ & 1 & & & & \\
$\mathrm{c}_{4}$ & 472 & $746^{* *}$ & $786^{*}$ & $698^{* *}$ & 1 & & & \\
$\mathrm{c}_{5}$ & $896^{* *}$ & $929^{* *}$ & $861^{* *}$ & $988^{* *}$ & 638 & 1 & & \\
$\mathrm{~b}_{1}$ & $940^{* * *}$ & $896^{* * *}$ & $839^{* *}$ & $884^{* * *}$ & 620 & $862^{* *}$ & 1 & \\
$\mathrm{~b}_{2}$ & $918^{* * *}$ & $930^{* *}$ & $870^{* * *}$ & $923^{* * *}$ & $671^{*}$ & $907^{* *}$ & $906^{* * *}$ & 1 \\
\hline
\end{tabular}

\section{3. 正規化 (Normalization)}

依據以上的數據如表 1 說明加以正規化, 如 (表4)。

表400分局2014年基本資料正規化資料。

\begin{tabular}{llllllll}
\hline & $\mathrm{c}_{1}$ & $\mathrm{c}_{2}$ & $\mathrm{c}_{3}$ & $\mathrm{c}_{4}$ & $\mathrm{c}_{5}$ & $\mathrm{~b}_{1}$ & $\mathrm{~b}_{2}$ \\
\hline $\mathrm{A}$ & 0.9789 & 0.6357 & 0.9612 & 153.2 & 0.1621 & 22 & 486.9 \\
$\mathrm{~B}$ & 0.9864 & 0.5579 & 0.9670 & 69.7 & 0.1535 & 25 & 476.6 \\
$\mathrm{C}$ & 0.9841 & 0.5255 & 0.9614 & 106.5 & 0.1457 & 25 & 422.3 \\
$\mathrm{D}$ & 0.9895 & 0.6977 & 0.9697 & 74.6 & 0.1213 & 14 & 263.8 \\
$\mathrm{E}$ & 0.9753 & 0.7198 & 0.9622 & 106.1 & 0.1741 & 16 & 226.9 \\
$\mathrm{~F}$ & 0.9778 & 0.7070 & 0.9290 & 169.3 & 0.3118 & 15 & 272.6 \\
$\mathrm{G}$ & 0.9898 & 0.8645 & 0.9842 & 81.6 & 0.0744 & 19 & 282.1 \\
$\mathrm{H}$ & 0.9915 & 0.7876 & 0.9847 & 97.2 & 0.0665 & 13 & 216.8 \\
$\mathrm{I}$ & 0.9874 & 0.5965 & 0.9737 & 46.0 & 0.1349 & 13 & 247.2 \\
\hline
\end{tabular}

\section{4. Delphi Technique求算權重}

本Delphi Technique先設計問卷，並以1-10為表示各 該單項重要性之分數值, 以上列五項產出為對象 (投入項 為固定因素, 不加權重), 調查對象為分局之2位副分局長, 6 位組長, 2 位隊長, 1 位主任, 計 11 人, 從警年資最高者 35 年, 最少者 20 年, 均深諳警察機關文化, 對警察工作極 為熟稔, 專業性及準確性, 均符合專家身分的要求; 依上 述方法公式 (4)、公式 (5) 運算, 計算各評估準則 $C_{1}, C_{2}, \ldots, C_{n}$ 之權重值 $w_{j}$ （如表 5 ）, 得:

$$
w_{j}=(0.2068,0.2165,0.1971,0.2117,0.1679)
$$

表5 警察機關工作指標權重調查統計表。

\begin{tabular}{llllll}
\hline & $\mathrm{C}_{1}$ & $\mathrm{C}_{2}$ & $\mathrm{C}_{3}$ & $\mathrm{C}_{4}$ & $\mathrm{C}_{5}$ \\
\hline 專家1 & 9 & 10 & 9 & 10 & 9 \\
專家2 & 6 & 8 & 9 & 8 & 6 \\
專家3 & 8 & 10 & 7 & 9 & 5 \\
專家4 & 8 & 7 & 9 & 10 & 5 \\
專家5 & 9 & 10 & 6 & 9 & 5 \\
專家6 & 7 & 8 & 5 & 6 & 8 \\
專家7 & 3 & 7 & 2 & 8 & 5 \\
專家8 & 10 & 10 & 10 & 10 & 5 \\
專家9 & 9 & 3 & 8 & 4 & 7 \\
專家10 & 9 & 9 & 8 & 6 & 7 \\
專家11 & 7 & 7 & 8 & 7 & 7 \\
e & 85 & 89 & 81 & 87 & 69 \\
W & 0.2068 & 0.2165 & 0.1971 & 0.2117 & 0.1679 \\
\hline
\end{tabular}

以總生產效率方法計算出相關生產力之比較, 總生產 效率等於產出與投入的比, 先以此概念基於投入固定, 再 加以對產出項先以主觀權重法 (Delphi) 對產出項做加權 後 (如表6) 求出其總生產力, 再以總生產力最佳之單位為 分母, 求出各DMU之相對效率, 並排序如下 (如表7), 並與 其他演算法做比較 (如結論說明)：

表6 對產出項主觀加權後資料。

\begin{tabular}{llllllll}
\hline DMU & $\mathrm{c}_{1}$ & $\mathrm{c}_{2}$ & $\mathrm{c}_{3}$ & $\mathrm{c}_{4}$ & $\mathrm{c}_{5}$ & $\mathrm{~b}_{1}$ & $\mathrm{~b}_{2}$ \\
\hline $\mathrm{A}$ & 0.2024 & 0.1377 & 0.1894 & 32.4292 & 0.0272 & 22.000 & 486.900 \\
$\mathrm{~B}$ & 0.2040 & 0.1208 & 0.1906 & 14.7540 & 0.0258 & 25.000 & 476.600 \\
$\mathrm{C}$ & 0.2035 & 0.1138 & 0.1895 & 22.5438 & 0.0245 & 25.000 & 422.300 \\
$\mathrm{D}$ & 0.2046 & 0.1511 & 0.1911 & 15.7912 & 0.0204 & 14.000 & 263.800 \\
$\mathrm{E}$ & 0.2017 & 0.1559 & 0.1896 & 22.4591 & 0.0292 & 16.000 & 226.900 \\
$\mathrm{~F}$ & 0.2022 & 0.1531 & 0.1831 & 35.8372 & 0.0523 & 15.000 & 272.600 \\
G & 0.2047 & 0.1872 & 0.1940 & 17.2730 & 0.0125 & 19.000 & 282.100 \\
H & 0.2051 & 0.1706 & 0.1941 & 20.5752 & 0.0112 & 13.000 & 216.800 \\
I & 0.2042 & 0.1292 & 0.1919 & 9.7372 & 0.0226 & 13.000 & 247.200 \\
\hline
\end{tabular}

表7 以產出項主觀加權後得之生產效率與DEA演算相對效率比較。

\begin{tabular}{|c|c|c|c|c|c|c|c|c|}
\hline & Delphi加權 & & & DEA演算 & & & 綜合主宾 & \\
\hline DMU & 總效率 & 相對效率 & 排序 & 技術效率 & 被參考次數 & 排序 & 平均值 & 排序 \\
\hline $\bar{A}$ & 0.0648 & 0.5117 & 4 & 0.67 & 0 & 7 & 0.5909 & 7 \\
\hline B & 0.0305 & 0.2407 & 9 & 0.532 & 0 & 9 & 0.3864 & 9 \\
\hline $\mathrm{C}$ & 0.0516 & 0.4073 & 7 & 0.553 & 0 & 8 & 0.4801 & 8 \\
\hline D & 0.0589 & 0.4649 & 6 & 0.946 & 0 & 5 & 0.7055 & 4 \\
\hline E & 0.0948 & 0.7487 & 2 & 1 & 2 & 3 & 0.8744 & 2 \\
\hline
\end{tabular}




\begin{tabular}{|c|c|c|c|c|c|c|c|c|}
\hline & Delphi加權 & & & DEA演算 & & & 綜合主宾 & \\
\hline DMU & 總效率 & 相對效率 & 排序 & 技術效率 & 被參考次數 & 排序 & 平均值 & 排序 \\
\hline F & 0.1267 & 1.0000 & 1 & 1 & 4 & 2 & 1.0000 & 1 \\
\hline G & 0.0594 & 0.4686 & 5 & 0.846 & 0 & 6 & 0.6573 & 5 \\
\hline H & 0.0921 & 0.7268 & 3 & 1 & 5 & 1 & 0.8634 & 3 \\
\hline I & 0.0395 & 0.3121 & 8 & 1 & 2 & 4 & 0.6560 & 6 \\
\hline
\end{tabular}

\section{5. 以DEAP2. 1演算所得相對效率}

將上述正規化後之資料以 $b_{1} 、 b_{2}$ 為投入項，分別 5 次 以 $c_{1} 、 c_{2} 、 c_{3} 、 c_{4} 、 c_{5}$ 各為產出項, 以DEAP軟體運算出相 對效率取scale為判斷指標。

\section{5. 1. 固定規模報酬 (CRS) 效率分析}

求固定規模報酬 (CRS) 作分析 (如表8), 以H、F、E、I 等四個派出所為相對有效率單位, 其中以大樹 $(\mathrm{H})$ 被相對 參考者為 5 次, 效率相對最佳, 大華 $(\mathrm{F}) 4$ 次居次。而相對 無效率的單位依序為D、G、A、C、B。

表8 效率及被參考次數分析。

\begin{tabular}{llllll}
\hline DMU & 技術效率 & 被參考次數 & 排序 & 參考對象 & 參考權重 \\
\hline 仁武 (A) & 0.670 & 0 & 7 & $\mathrm{H}, \mathrm{F}$ & $0.264,0.753$ \\
澄觀 (B) & 0.532 & 0 & 9 & $\mathrm{H}, \mathrm{I}, \mathrm{F}$ & $0.746,0.148,0.106$ \\
大社 (C) & 0.553 & 0 & 8 & $\mathrm{~F}, \mathrm{H}, \mathrm{E}$ & $0.285,0.086,0.628$ \\
仁美 (D) & 0.946 & 0 & 5 & $\mathrm{I}, \mathrm{H}, \mathrm{F}$ & $0.447,0.462,0.095$ \\
鳥松 (E) & 1.000 & 2 & 3 & $\mathrm{E}$ & 1.000 \\
大華 (F) & 1.000 & 4 & 2 & $\mathrm{~F}$ & 1.000 \\
九曲 (G) & 0.846 & 0 & 6 & $\mathrm{E}, \mathrm{H}$ & $0.003,1.098$ \\
大樹 (H) & 1.000 & 5 & 1 & $\mathrm{H}$ & 1.000 \\
溪埔 (I) & 1.000 & 2 & 4 & $\mathrm{I}$ & 1.000 \\
\hline
\end{tabular}

\section{5. 2. 差額變數分析 (SUMMARY OF OUTPUT SLACKS)}

從管理的角度, DEA模式優點, 除了解 DMU 無效率原 因外，並訂定改善策略（或改善量），差額變數分析主要 目的，在於提供相對效率小於 1 的DMU目前資源使用情形, 再透過瞭解其投入資源與產出數量存在多少改善空間, 以 作為管理者之經營目標設定的參考。相對效率差的單位經 分析在DMU-A而言, 在投入方面可減少油料的支出, 而產 出方面, 減少全般刑案發生率、增加全般刑案破獲率及 110 受理案件的處理等方面, 在DMU-B而言, 在投入方面可減 少油料的支出, 而產出方面, 增加全般刑案破獲率等方面。
在DMU-C而言, 在投入方面不需變動, 而產出方面, 增加 全般刑案破獲率、減少全般車禍發生率及增加交通違規取 締數等方面。在DMU-D而言, 在投入方面可減少油料的支 出, 而產出方面, 減少全般車禍發生率及增加交通違規 取締數等方面。在DMU-G而言, 在投入方面可減少警力數, 而產出方面, 減少全般刑案發生率、以減少全般車禍發生 率及增加交通違規取締數方面 (如表9)。而各單位應改進 之各項投入或產出之比率或件 (個) 數, 詳如表 10 、表 11 所列), 可供各DMU在決策時的參考, 極具價值。

表9 產出及投入差額分析。

\begin{tabular}{|c|c|c|c|c|c|c|c|}
\hline \multirow[t]{2}{*}{ DMU } & \multicolumn{5}{|c|}{ (Output Slacks) 產出差額 } & \multicolumn{2}{|c|}{ (Input Slacks) 投入差額 } \\
\hline & $\mathrm{c}_{1}$ & $\mathrm{c}_{2}$ & $\mathrm{c}_{3}$ & $\mathrm{c}_{4}$ & $\mathrm{c}_{5}$ & $b_{1}$ & $\mathrm{~b}_{2}$ \\
\hline 仁武 (A) & 0.003 & 0.019 & 0.000 & 0.000 & 0.231 & 0.000 & 63.456 \\
\hline 澄觀 (B) & 0.000 & 0.013 & 0.000 & 0.000 & 0.000 & 0.000 & 5.747 \\
\hline 大社 (C) & 0.000 & 0.041 & 0.001 & 2. 080 & 0.000 & 0.000 & 0.000 \\
\hline 仁美 (D) & 0.000 & 0.000 & 0.002 & 1. 213 & 0.000 & 0.000 & 12.952 \\
\hline 鳥松 (E) & 0.000 & 0.000 & 0.000 & 0.000 & 0.000 & 0.000 & 0.000 \\
\hline 大華 (F) & 0.000 & 0.000 & 0.000 & 0.000 & 0.000 & 0.000 & 0.000 \\
\hline 大樹 (H) & 0.000 & 0.000 & 0.000 & 0.000 & 0.000 & 0.000 & 0.000 \\
\hline 溪埔 (I) & 0.000 & 0.000 & 0.000 & 0.000 & 0.000 & 0.000 & 0.000 \\
\hline
\end{tabular}

表10 產出及投入目標分析。

\begin{tabular}{lllllll}
\hline DMU & \multicolumn{2}{l}{ (Output } & Targets) 產出目標 & & & (Input Targets) 投入目標 \\
\hline & $\mathrm{c}_{1}$ & $\mathrm{c}_{2}$ & $\mathrm{c}_{3}$ & $\mathrm{c}_{4}$ & $\mathrm{c}_{5}$ & $\mathrm{~b}_{1}$ \\
\hline 仁武(A) & 0.170 & 0.132 & 0.156 & 26.710 & 0.253 & 14.732 \\
澄觀 (B) & 0.168 & 0.113 & 0.157 & 12.152 & 0.154 & 13.299 \\
大社 (C) & 0.168 & 0.135 & 0.157 & 20.648 & 0.146 & 13.815 \\
仁美 (D) & 0.169 & 0.124 & 0.159 & 14.220 & 0.121 & 13.240 \\
鳥松 (E) & 0.166 & 0.128 & 0.156 & 18.499 & 0.174 & 233.362 \\
大華 (F) & 0.167 & 0.126 & 0.151 & 29.517 & 0.312 & 16.000 \\
\hline
\end{tabular}




\begin{tabular}{llllllll}
\hline DMU & \multicolumn{2}{l}{ (Output Targets) 產出目標 } & & & & (Input Targets) 投入目標 \\
\hline & $\mathrm{c}_{1}$ & $\mathrm{c}_{2}$ & $\mathrm{c}_{3}$ & $\mathrm{c}_{4}$ & $\mathrm{c}_{5}$ & $\mathrm{~b}_{1}$ & $\mathrm{~b}_{2}$ \\
\hline 九曲 (G) & 0.186 & 0.154 & 0.176 & 18.650 & 0.074 & 14.311 & 238.556 \\
大樹(H) & 0.169 & 0.140 & 0.160 & 16.947 & 0.067 & 13.000 & 216.800 \\
溪埔 (I) & 0.168 & 0.106 & 0.158 & 8.020 & 0.135 & 13.000 & 247.200 \\
\hline
\end{tabular}

表11 各DMU之改進值。

\begin{tabular}{llllllll}
\hline & $\mathrm{c}_{1}$ & $\mathrm{c}_{2}$ & $\mathrm{c}_{3}$ & $\mathrm{c}_{4}$ & $\mathrm{c}_{5}$ & $\mathrm{~b}_{1}$ & $\mathrm{~b}_{2}$ \\
\hline 仁武 (A) & 0.513 & 0.471 & 0.473 & 75.371 & 0.214 & 0.000 & -94.839 \\
澄觀 (B) & 0.869 & 0.633 & 0.854 & 61.376 & 0.136 & 0.000 & -10.917 \\
大社(C) & 0.798 & 0.846 & 0.786 & 107.874 & 0.118 & 0.000 & 0.000 \\
仁美 (D) & 0.059 & 0.041 & 0.063 & 12.861 & 0.007 & 0.000 & -13.976 \\
鳥松(E) & 0.000 & 0.000 & 0.000 & 0.000 & 0.000 & 0.000 & 0.000 \\
大華 (F) & 0.000 & 0.000 & 0.000 & 0.000 & 0.000 & 0.000 & 0.000 \\
九曲 (G) & 0.299 & 0.159 & 0.296 & 44.941 & 0.014 & -2.049 & 0.000 \\
大樹(H) & -0.001 & 0.000 & 0.000 & 0.000 & -0.001 & 0.000 & 0.000 \\
溪埔 (I) & 0.000 & -0.001 & 0.000 & 0.000 & 0.000 & 0.000 & 0.000 \\
\hline
\end{tabular}

表12 以VRS分析資料結果表。

\begin{tabular}{llllll}
\hline & 技術效率 (綜合) & 純技術效率 & 規模效率 & 規模報酬 & 標等學習對象 \\
\hline 仁武 (A) & 0.670 & 1.000 & 0.670 & $\mathrm{drs}$ & $\mathrm{H}, \mathrm{I}, \mathrm{F}$ \\
澄觀 (B) & 0.532 & 0.534 & 0.996 & $\mathrm{drs}$ & $\mathrm{E}, \mathrm{H}, \mathrm{F}$ \\
大社 (C) & 0.553 & 0.553 & 0.999 & - & \\
仁美 (D) & 0.946 & 1.000 & 0.946 & $\mathrm{drs}$ & \\
鳥松 (E) & 1.000 & 1.000 & 1.000 & - & \\
大華 (F) & 1.000 & 1.000 & 1.000 & - & \\
九曲 (G) & 0.846 & 1.000 & 0.846 & $\mathrm{drs}$ & \\
大樹 (H) & 1.000 & 1.000 & 1.000 & - & - \\
溪埔 (I) & 1.000 & 1.000 & 1.000 & - & \\
\hline
\end{tabular}

本研究另以「變動規模報酬」（variable return to scale, VRS)BCC模式分析相關數據, 得純技術效率方面, 除 $B, C$ 等 2 單位外, 都相對有效率, 而 $B, C$ 等 2 單位可向 $H$, I, F及E，H，F等單位學習，而其中B單位為相對無效率單 位, 卻又是規模報酬遞減 $(d r s)$ 現象, 每增加一個單位的 投入，其產出不足一個單位，故其在工作的技術方面，有 很大的改進空間 (如表12)。

\section{5. 結論與建議}

本研究以DEA分析法建立警政績效評估模式，評估各 派出所執行工作效率之優劣次序, 可供警政機關便於瞭解 各派出所執行績效是否相對有效率。因DEA績效指標權重 為演算法中自行設定, 屬客觀權重法之一種, 但為免與現 實不符, 故以Delphi主觀權重法另為演算, 以為比較, 發 現稍有些微變化。5個準則權重排序, 其中以全般刑案破 獲率 $\left(\mathrm{w}_{2}\right)$ 最高, 符合實務上破案為重的現象, 110 受理案 件平均數 $\left(\mathrm{w}_{5}\right)$ 最小。由此可知, 全般刑案破獲率 $\left(\mathrm{w}_{2}\right)$ 對 於受評估之派出所工作績效是極具重要性的指標, 也是現 階段警政工作對績效要求的偏向。

另外, 2014年樣本派出所中, 以主觀權重法加以演算 並排序結果, 以F為最佳單位, B為相對較差單位; 另以DEA 演算法分析, 則E、F、H、I 同為績效為 1 之績優派出所, 但再比較被參考次數, 則以 $\mathrm{H}$ 為佳, 但相對最無效率, 仍 為B派出所, 若將主客觀加以平均則排序結果以 $\mathrm{F}$ 為最佳單 位, B為營運績效相對較差。此類模式, 對於警政單位之
績效評估，應有相當之參考價值。至於相對績效較差單位 如何改進作為, 提升效率已於 4.5 節說明, 不再贅述。

若警察機關對於績效評估仍需延襲使用業績管理系 統 (PBM)，即所稱之目標管理，需先設定績效目標，評估 達成率以為評核基準, 本文建議可提出數項評估準則後, 再以本法排定各受評單位的繁簡程度, 再依序設定各單位 應達成的目標值, 使各單位的績效評核基準, 更符合實際。

\section{参考文献}

[1] Xing, Tai-Ping, Huang, Zheng-Zhi, Zeng, Guo-Xiong. (2001). A Model for Productivity Assessment of Criminal Investigation and Crime Prevention in Taiwan Police Departments: A Malmquist Output Index Approach. Journal of Information, Technology And Society, 17-40.

[2] Chi, Hsin-yi. (2007). Police Force Investment and Relative Crime Prevention Efficiency in Taiwan: The Application of Data Envelopment Analysis, academic costumes.

[3] Kuo-Chan Chang. (2013). The Case Study of Public Order Performance: the Effect of Pre- and Post-mergers on Criminal Cases of Kaohsiung City, doctor's degree costumes. 
［4］吳濟華、何柏正 (2008). 組織效率與生產力評估：資料包絡 分析法, 前程文化出版。

[5] Tien-Chin Wang and Jo-Chien Hsu. (2004). Evaluation of the Business 0peration Performance of the Listing Companies by Applying TOPSIS Method. International Conference on Systems, Man and Cybernetics (IEEE SMC 2004). the Hague, the Netherlands, 1286-1291.

[6] Webb Natalie and Angelis Diane. (2009). Improving Performance Measurement in Defense Organizations. Armed Forces Comptroller, 54(1), 16-21.

[7] Minder, Stephen W., Leinicke, Linda M., Rexroad, W. Max. (2014). What Do You Measure? Internal Auditor, $71(2), \quad 63-67$.

[8] Xiao-Ya Li. (2015). Employing post-dea method in budget management of health care sectors. 12th International Symposium on Operations Research and its Applications in Engineering, Technology and Management (ISORA 2015), 1-6.

[9] Melissa A. Schilling. (2017). Strategic management of technological innovation. fifth edition, 147-149.

[10] Zi jiang Yang, Guangzao Huang, Xing Tan. (2015). DEA evaluation of health project performance. 2015 IEEE International Conference on Progress in Informatics and Computing (PIC), 534-538.

[11] G. R. Jahanshahloo, F. Hosseinzadeh Lotfi, Y. Jafari, R. Maddahi. (2011). Selecting symmetric weights as a secondary goal in DEA cross-efficiency evaluation. Applied Mathematical Modelling, 35(1), 544-549.

[12] Si-Fong Jhang and Ci-Wu Jhou. (1992). Data Envelopment Analysis on efficiency evaluation of application. The Chinese Accounting Review, 26, 76-99.

[13] Hsiang-Jung Tsai and Hung-kuang Chang. (1998). An Evaluation and Selection of Weighting Techniques. The sixth defense management academic and practice Symposium, Taipei: National Defense Management Institute.

[14] Chn-Chun Liu. (1998). Public Sector Efficiency Measurement-Application of DEA and AHP. Web Journal of Chinese Management Review, 1(2).
[15] Wade D. Cook and Joe Zhu. (2015). DEA Cross Efficiency, Volume 221 of the series International Series in Operations Research \& Management Science, 23-43.

[16] Ching-Kuo Wei, Rong-Kwei Li and Liang-Chih Chen. (2011). The Study on Optimal Weight of Data Envelopment Analysis: A Case Study of Taiwan Medical Center. Journal of Management and Systems, 18(3), 505-527.

[17] Tien-Chin Wang and Hsien-Da Lee. (2009). Developing a Fuzzy TOPSIS Approach Based on Subjective Weights and Objective Weights. Expert Systems with Applications, 36, 8980-8985.

[18] Chn-Chun Liu. (2004). A study of weight restrict in data envelopment analysis. Chung Hua Journal of Management, 5(2), 93-104.

[19] Boghossian, Peter; Myers, Paula; Kilmer, Gary. (2010). The Delphi Technique: Correctional Administration and Community Consensus. Corrections Today, 72(4), 71-73.

[20] Coelli T. J. A Guide to DEAP Version 2. 1: A Data Envelopment Analysis (Computer) Program Centre for Efficiency and Productivity Analysis. (CEPA) Working Papers, No. 8/96.

[21] Wang Hong Li, Liang Liang, Wade D. Cook, and Joe Zhu. (2016). DEA models for non-homogeneous DMUs with different input configurations. European Journal of Operational Research, 254(3), 946-956.

[22] Zeng Guo Xiong and Xing Tai Ping. (2002). Accessing Efficiency of Criminal Investigation and Crime Prevention in Taiwan Police Department: An Application of DEA and AHP. Journal of Information Technology and Society, 33-56.

[23] 鄭信德 (2011). 資源回收績效之探討一以各縣市政府推動 成果為例, 臺灣銀行季刊第六十二卷第四期, 70-99。

[24] 徐中琦、許志忠 (2011). 台灣壽險業經營效率之研究-資料 包絡分析法之應用，臺灣銀行季刊第六十二卷第二 期, 42-67。 